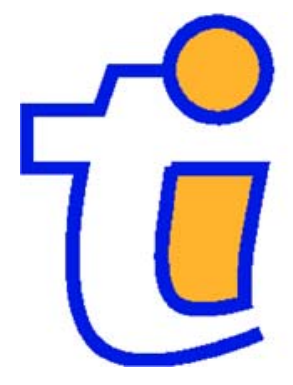

TI 2009-108/1

Tinbergen Institute Discussion Paper

Comparable Axiomatizations of the Myerson Value, the Restricted Banzhaf Value, Hierarchical Outcomes and the Average Tree Solution for Cycle-Free Graph Restricted Games

René van den Brink 


\section{Tinbergen Institute}

The Tinbergen Institute is the institute for economic research of the Erasmus Universiteit Rotterdam, Universiteit van Amsterdam, and Vrije Universiteit Amsterdam.

Tinbergen Institute Amsterdam

Roetersstraat 31

1018 WB Amsterdam

The Netherlands

Tel.: +31(0)205513500

Fax: $+31(0) 205513555$

Tinbergen Institute Rotterdam

Burg. Oudlaan 50

3062 PA Rotterdam

The Netherlands

Tel.: + $31(0) 104088900$

Fax: $+31(0) 104089031$

Most TI discussion papers can be downloaded at http://www.tinbergen.nl. 


\title{
Comparable Axiomatizations of the Myerson Value, the Restricted Banzhaf Value, Hierarchical Outcomes and the Average Tree Solution for Cycle-Free Graph Restricted Games*
}

\author{
René van den Brink ${ }^{\dagger}$
}

November 24, 2009

*Preliminary version. This research is part of the Research Program "Strategic and Cooperative Decision Making".

${ }^{\dagger}$ J.R. van den Brink, Department of Econometrics and Tinbergen Institute, VU University, De Boelelaan 1105, 1081 HV Amsterdam, The Netherlands. E-mail: jrbrink@feweb.vu.nl 


\begin{abstract}
We consider cooperative transferable utility games, or simply TU-games, with a limited communication structure in which players can cooperate if and only if they are connected in the communication graph. A difference between the restricted Banzhaf value and the Myerson value (i.e. the Shapley value of the restricted game) is that the restricted Banzhaf value satisfies collusion neutrality, while the Myerson value satisfies component efficiency. Requiring both efficiency and collusion neutrality for cycle-free graph games yields other solutions such as the hierarchical outcomes and the average tree solution. Since these solutions also satisfy the superfluous player property, this also 'solves' an impossibility for TU-games since there is no solution for these games that satisfies efficiency, collusion neutrality and the null player property.

We give axiomatizations of the restricted Banzhaf value, the hierarchical outcomes and the average tree solution that are comparable with axiomatizations of the Myerson value in case the communication graph is cycle-free. Finally, we generalize these solutions to classes of solutions for cycle-free graph games using network power measures.
\end{abstract}

Keywords: Cooperative TU-game, communication structure, Myerson value, Shapley value, Banzhaf value, hierarchical outcome, average tree solution, component efficiency, collusion neutrality.

JEL code: C71 


\section{Introduction}

A situation in which a finite set of players can obtain certain payoffs by cooperation can be described by a cooperative game with transferable utility, or simply a TU-game, being a pair $(N, v)$, where $N=\{1, \ldots, n\}$ is a finite set of players and $v: 2^{N} \rightarrow \mathbb{R}$ is a characteristic function on $N$ such that $v(\emptyset)=0$. For any coalition $S \subseteq N, v(S)$ is the worth of coalition $S$, i.e., the members of coalition $S$ can obtain a total payoff of $v(S)$ by agreeing to cooperate.

In a TU-game any subset $S \subseteq N$ is assumed to be able to form a coalition and earn the worth $v(S)$. However, in most economic and political organizations not every set of participants can form a feasible coalition. One of the most well-known restrictions in cooperation are the games with limited communication structure as introduced by Myerson (1977) in which the members of some coalition $S$ can realize the worth $v(S)$ if and only if they are connected within a given communication graph on the set of players. Solutions for graph-restricted games studied in the literature are, a.o. the Myerson value (see Myerson (1977)), the position value (see Borm, Owen and Tijs (1992)) and recently, for cyclefree graph games the hierarchical outcomes (see Demange (2004)) and their average (see Herings, van der Laan and Talman (2008)). The hierarchical outcomes are introduced in Demange (2004) and are extreme points of the Core of the Myerson restricted game in case the game $v$ is superadditive and the communication graph is a tree. This definition can be extended to cycle-free graph games in a straightforward way by defining the payoffs componentwise. This implies that for these graph games also all convex combinations, in particular the average, of the hierarchical outcomes belong to the Core of the restricted game. The solution that assigns to every cycle-free graph game the average hierarchical outcome is introduced in Herings, van der Laan and Talman (2008) as the average tree solution.

Both the Myerson value as well as the average tree solution are characterized by component efficiency (stating that every component in the communication graph earns exactly its own worth) and some fairness or equal gain/loss axiom (equalizing the changes in payoffs of certain players after deleting links from the communication graph), see Myerson (1977), respectively, Herings, van der Laan and Talman (2008). Alternatively, Borm, Owen and Tijs (1992) provide axiomatizations of the Myerson value and the position value for cycle-free graph games using component efficiency, additivity, the superfluous link property (stating that deleting so-called superfluous links does not change the payoff distribution) and the communication ability property (stating that the players earn equal payoffs in case they are in some sense equal in the communication graph game) $)^{1}$.

\footnotetext{
${ }^{1}$ In fact, they apply the communication ability property only to characterize the Myerson value, while they use the degree property to characterize the position value. But, as shown by van den Brink, van der Laan and Pruzhansky (2004), both solutions can be characterized by a communication ability property
} 
It turns out that this axiomatization is very useful in comparing the Myerson value with the restricted Banzhaf value (i.e. applying the Banzhaf value to the restricted game) and the average tree solution for cycle-free graph games. From the axioms mentioned above, the restricted Banzhaf value does not satisfy component efficiency. Instead it satisfies a variation of the collusion neutrality axioms of Haller (1994) and Malawski (2002) for TUgames. These collusion neutrality axioms state that, if two players in a TU-game collude and act as if they are one player, then the sum of their payoffs should not change. For TU-games, both efficiency (stating that the sum of payoffs over all players equals the worth of the 'grand coalition' $N$ ) and collusion neutrality seem to be desirable properties of which the Shapley value satisfies efficiency but not collusion neutrality, and the Banzhaf value satisfies collusion neutrality but not efficiency. In van den Brink (2009) it is shown that there is no solution for TU-games that satisfies efficiency, collusion neutrality and the null player property ${ }^{2}$ (stating that a null player in a game earns a zero payoff). Thus, requiring efficiency and collusion neutrality to be satisfied at the same time is very restrictive for TU-games.

When considering graph restricted games we might also apply the communication restrictions to certain axioms. For example, efficiency is usually strengthened to component efficiency. On the other hand, it seems reasonable to weaken collusion neutrality by allowing only neighbours in the communication graph to collude. Indeed, it seems counterintuitive that two players who cannot cooperate because they cannot communicate, would be able to collude. The restricted Banzhaf value satisfies this collusion neutrality and together with additivity, the superfluous link property, the communication ability property and the isolated player property (stating that players that have no neighbours earn their own worth) characterizes this solution.

Of course, the restricted Banzhaf value does not satisfy component efficiency. As mentioned above, for TU-games there is no solution satisfying efficiency, collusion neutrality and the null player property. However, for cycle-free graph games it turns out that all hierarchical outcomes ${ }^{3}$ and their convex combinations ${ }^{4}$ (thus also the average tree solution) satisfy component efficiency, collusion neutrality and the superfluous player property

and a degree measure property, by taking the appropriate power measure for communication graphs. In particular, the equal power measure for the Myerson value and the degree measure for the so-called Harsanyi degree solution (wich is equal to the position value on cycle-free graph games and is equal to the Shapley value on complete graph games).

${ }^{2}$ There it is also shown that the equal division solution, being the solution that distributes $v(N)$ equally over all players, is the only solution for TU-games satisfying efficiency, collusion neutrality and symmetry.

${ }^{3}$ Note that a hierarchical outcome is a payoff vector. If no confusion arises, in the sequel we will refer also to the solution that assigns to every cycle-free graph game this payoff vector as a hierarchical outcome.

${ }^{4}$ These convex combinations are the random tree solutions as considered in Béal, Rémila and Solal (2009). 
(stating that a null player in the restricted game earns a zero payoff). Therefore, the 'impossibility' concerning the existence of a solution for TU-games satisfying these axioms is 'solved' when restricting cooperation by allowing only connected coalitions in a cycle-free communication graph to cooperate and neighbours to collude ${ }^{5}$. Adding the communication ability property, the equal gain/loss property (stating that after a collusion of two players, the payoffs of other players change by the same amount) and component independence characterizes the average tree solution.

From the axioms mentioned above, the Myerson value and the Banzhaf value do not satisfy the equal gain/loss property (besides component efficiency, respectively, collusion neutrality). However, for the axiomatization of the average tree solution it is sufficient to require the weaker axiom which states that after a collusion between two neighbouring players that are symmetric in the restricted game, the payoffs of other players in the same component that are symmetric with them, change by the same amount. In this way the Myerson value, the restricted Banzhaf value and the average tree solution are characterized by comparable axioms, where the Myerson value and the average tree solution have in common that they both satisfy component efficiency, the average tree solution and the restricted Banzhaf value have in common that they both satisfy collusion neutrality, and the Myerson value and the restricted Banzhaf value have in common that they both satisfy the superfluous link property.

It is also clear that the average tree solution satisfying both component efficiency and collusion neutrality goes 'at a cost', being that it does not satisfy the superfluous link property. But similar disadvantages can be mentioned for the Myerson value (not satisfying collusion neutrality) and restricted Banzhaf value (not satisfying component efficiency).

Finally, we can easily generalize the above mentioned axiomatizations of the restricted Banzhaf value and the average tree solution in a similar way as done in van den Brink, van der Laan and Pruzhansky (2004) for the Myerson value, by generalizing the communication ability property to the $\sigma$-communication ability property. For any positive network power measure ${ }^{6} \sigma$ this axiom states that in case the players are in some sense equal in the communication graph game, then the (Harsanyi) dividends (see Harsanyi (1959)) are allocated proportional to the power of the players in the corresponding coalitions. Ob-

\footnotetext{
${ }^{5}$ A well-known result for cycle-free graph games is given by Le Breton, Owen and Weber (1992) and Demange (1994) who showed that these communication restrictions guarantee the existence of a Core payoff vector in the restricted game when $v$ is superadditive. Demange (2004) introduced the hierarchical outcomes as (some) extreme points of this Core.

${ }^{6} \mathrm{~A}$ network power measure is a function that assigns to every node in any communication graph a real number that can be seen as a measure of the 'power' or 'influence' of that node in the communication graph. It is positive if it assigns a positive value to every node that has at least one neighbour, and zero to isolated nodes that have no neighbour.
} 
viously, taking the equal power measure that assigns to every connected player the same power (and isolated players having power zero) yields the communication ability property. For trees (i.e. connected, cycle-free graphs) with a fixed root player $i$, taking as power measure the one that assigns in any connected coalition full power to the player that is 'closest' to the root player $i$ and assigns power zero to the other players yields (together with the other axioms used to characterize the average tree solution) an axiomatization of the corresponding hierarchical outcome with root player $i$. Another solution is obtained when taking the degree measure as power measure which, in the axiomatic system of Borm, Owen and Tijs (1992), yields the position value. In this way we obtain some kind of average tree-position value and restricted Banzhaf-position value. (Note that the position value itself does not satisfy collusion neutrality.)

This paper is organized as follows. Section 2 contains preliminaries on TU-games, communication graphs and communication graph games. In Section 3 we provide an axiomatization of the restricted Banzhaf value for cycle-free graph games. In Section 4 we axiomatize the average tree solution for cycle-free graph games. In Section 5 we consider generalizations of the restricted Banzhaf value and average tree solution (including the hierarchical outcomes) using network power measures. Finally, Section 6 contains concluding remarks.

\section{Preliminaries}

\subsection{Cooperative TU-games}

Since in this paper we take the player set $N$ to be fixed, we represent a TU-game $(N, v)$ just by its characteristic function $v$. The collection of all TU-games on $N$ (represented by their characteristic function) is denoted by $\mathcal{G}^{N}$.

A special class of games are the unanimity games. The unanimity game of coalition $T \subseteq N, T \neq \emptyset$, is the game $u_{T} \in \mathcal{G}^{N}$ given by $u_{T}(S)=1$ if $T \subseteq S$, and $u_{T}(S)=0$ otherwise. It is well-known that the unanimity games form a base of $\mathcal{G}^{N}$. Every game $v \in \mathcal{G}^{N}$ can be written as a linear combination of unanimity games $v=\sum_{\substack{T \subseteq N \\ T \neq \emptyset}} \Delta_{v}(T) u_{T}$ with $\Delta_{v}(T)=\sum_{S \subseteq T}(-1)^{|T|-|S|} v(S)$ being the Harsanyi dividends, see Harsanyi (1959).

A TU-game $(N, v)$ is superadditive if $v(S \cup T) \geq v(S)+v(T)$ for all $S, T \subseteq N$ with $S \cap T=\emptyset$, i.e. when two disjoint coalitions cooperate in a superadditive game then it is always possible to reallocate the worth of these coalitions in such a way that all players in the coalition benefit (or at least are not worse off) from the cooperation. We denote the collection of all superadditive TU-games on $N$ by $\mathcal{G}_{S}^{N}$.

A payoff vector of a game $v$ is an $n$-dimensional vector $x \in \mathbb{R}^{N}$ giving a payoff $x_{i} \in \mathbb{R}$ to player $i \in N$. A payoff vector $x$ is efficient for game $v$ if it exactly distributes 
the worth $v(N)$ of the 'grand coalition' $N$, i.e., $\sum_{i \in N} x_{i}=v(N)$. A (single-valued) solution for TU-games is a function $f$ that assigns to every game $v$ a payoff vector $f(v) \in \mathbb{R}^{N}$. A solution $f$ is efficient if $f(v)$ is efficient for every game $v$. The most famous efficient (singlevalued) solution is the Shapley value (Shapley, 1953) being the solution $S h: \mathcal{G}^{N} \rightarrow \mathbb{R}^{N}$ that assigns to every player its expected marginal contribution to the coalition of players that enter before that player, given that every order of entrance $\pi$ on $N$ has equal probability $\frac{1}{n !}$ to occur, i.e.

$$
S h_{i}(v)=\frac{1}{n !} \sum_{\pi \in \Pi(N)} m_{i}^{\pi}(v) \text { for all } i \in N,
$$

where $\Pi(N)$ is the collection of all permutations of $N$ and for $\pi \in \Pi(N)$,

$$
m_{i}^{\pi}(v)=v(\{j \in N \mid \pi(j) \leq \pi(i)\})-v(\{j \in N \mid \pi(j)<\pi(i)\})
$$

is the marginal contribution of player $i$ to the coalition of all its predecessors in permutation $\pi$.

The non-efficient solution that got most attention in the literature is the Banzhaf value (introduced by Banzhaf (1965) to measure voting power in voting games and generalized by Owen (1975) and Dubey and Shapley (1979) to general TU-games) being the solution $B a: \mathcal{G}^{N} \rightarrow \mathbb{R}^{N}$ that assigns to every player its expected marginal contribution given that every combination of the other players has equal probability of being the coalition that is already present when that player enters. Thus, it assigns to every player in a game its average marginal contribution, i.e.

$$
B a_{i}(v)=\frac{1}{2^{n-1}} \sum_{S \subseteq N \backslash\{i\}} m_{i}^{S}(v) \text { for all } i \in N,
$$

where $m_{i}^{S}(v)=v(S \cup\{i\})-v(S)$.

Using the Harsanyi dividends it is also known that the Shapley value and Banzhaf value can be written as

$$
S h_{i}(v)=\sum_{\substack{T \subseteq N \\ i \in T}} \frac{\Delta_{v}(T)}{|T|} \text {, respectively, } B a_{i}(v)=\sum_{\substack{T \subseteq N \\ i \in T}} \frac{\Delta_{v}(T)}{2^{|T|-1}} \text {, for all } i \in N .
$$

A set-valued solution for TU-games is a mapping $F$ that assigns to every game $v$ a set of payoff vectors $F(v) \subset \mathbb{R}^{N}$. A set-valued solution $F$ is efficient if all $x \in F(v)$ are efficient for every game $v$. The most famous set-valued solution is the Core (Gillies, 1953) that assigns to every game $v$ the set of all efficient and coalitionally stable payoff vectors, i.e.,

$$
\operatorname{Core}(v)=\left\{x \in \mathbb{R}^{n} \mid \sum_{i \in N} x_{i}=v(N) \text { and } \sum_{i \in S} x_{i} \geq v(S) \text { for all } S \subset N\right\} .
$$


For general TU-games, a Core payoff vector need not exist, even not for superadditive games $^{7}$.

\subsection{Graphs}

An undirected graph is a pair $(N, L)$ where $N$ is the set of nodes and $L \subseteq\{\{i, j\} \mid i, j \in$ $N, i \neq j$ \} is a collection of subsets of $N$ such that each element of $L$ contains precisely two nodes. The elements of $L$ represent bilateral communication links and are refered to as edges or links. Since in this paper the nodes in a graph represent the positions of players in a communication network we refer to the nodes as players. If there is a link between two players then we call them neighbours and we denote the set of neighbours of player $i$ by $R_{(N, L)}(i)=\{j \in N \mid\{i, j\} \in L\}$. A sequence of $k$ different players $\left(i_{1}, \ldots, i_{k}\right)$ is a path in $(N, L)$ if $\left\{i_{h}, i_{h+1}\right\} \in L$ for all $h=1, \ldots, k-1$. Two distinct players $i$ and $j, i \neq j$, are connected in graph $(N, L)$ if there is a path $\left(i_{1}, \ldots, i_{k}\right)$ with $i_{1}=i$ and $i_{k}=j$. A graph $(N, L)$ is connected if any two players $i, j \in N$ are connected. For some $S \subseteq N$, the graph $(S, L(S))$ with $L(S)=\{l \in L \mid l \subseteq S\}$ is called a subgraph of $(N, L)$. For given graph $(N, L)$, a set of players $S$ is connected when the subgraph $(S, L(S))$ is connected.

A subset $K$ of $N$ is a component in $(N, L)$ if the subgraph $(K, L(K))$ is maximally connected in $(N, L)$, i.e., the subgraph $(K, L(K))$ is connected, and for any $j \in N \backslash K$, the subgraph $(K \cup\{j\}, L(K \cup\{j\}))$ is not connected in $(N, L)$. For every coalition $S \subseteq N$, the components in $(S, L(S))$ form a partition of $S$. For graph $(N, L)$ and coalition $S \subseteq N$ we denote by $C_{L}(S)$ the set of all connected coalitions in $(S, L(S))$, and by $C_{L}^{m}(S)$ the partition of coalition $S$ into components of $(S, L(S))$. For $i \in N, K_{L}^{i}$ denotes the component of $N$ in $(N, L)$ to which $i$ belongs.

A path $\left(i_{1}, \ldots, i_{k}\right), k \geq 3$, is a cycle in $(N, L)$ if $\left\{i_{k}, i_{1}\right\} \in L$. A graph $(N, L)$ is cycle-free when it does not contain any cycle. A player $i \in N$ is called a pending player if it is connected to exactly one other player, i.e., if $|\{l \in L \mid i \in l\}|=1$. Note that a cycle-free communication graph $(N, L)$ with $|N| \geq 2$ and $L \neq \emptyset$ has at least two pending players. A graph that is connected and cycle-free is called a tree. We denote by $\mathcal{L}^{N}$ the collection of all sets of links $L$ such that $(N, L)$ is a graph on $N$. By $\mathcal{L}_{C F}^{N}$ we denote the collection of all sets of links $L$ such that $(N, L)$ is a cycle-free graph on $N$.

Finally, we denote by $D(N, L)=\left\{i \in N \mid R_{(N, L)}(i) \neq \emptyset\right\}$ the set of all non-isolated players in $(N, L)$.

\footnotetext{
${ }^{7}$ It is well-known that the Core of a game is nonempty if and only if the game is balanced, see Bondareva (1963) and Shapley (1967).
} 


\subsection{Graph games and solutions}

In a TU-game any subset $S \subseteq N$ is assumed to be able to form a coalition and earn the worth $v(S)$. However, in most economic and political organizations not every set of participants can form a feasible coalition. One of the most well-known restrictions on coalition formation are communication restrictions as introduced in Myerson (1977). In this model there is a communication network on the set of players in a cooperative game, and a coalition is feasible if and only if the players in this coalition are connected within this communication network. The communication network is represented as an undirected graph $(N, L)$ on the set of players $N$, where $L$ is the set of binary communication links between the players. A game $v \in \mathcal{G}^{N}$ with communication graph $(N, L)$ is shortly denoted by $(v, L)$ and referred to as a graph game. A (single-valued) solution for graph games is a function $f$ that assigns a payoff vector $f(v, L) \in \mathbb{R}^{N}$ to every graph game $(v, L)$ on $N$.

In the graph game $(v, L)$ the players in a coalition $S$ can cooperate and earn their worth $v(S)$ if and only if $S$ is connected in $(N, L)$. If $S$ is not connected we follow Myerson (1977) and assume that the players in $S$ can only realize the sum of the worths of the components of $S$. This yields Myerson (1977)'s restricted game $v^{L}$ given by

$$
v^{L}(S)=\sum_{T \in C_{L}^{m}(S)} v(T), \quad S \subseteq N,
$$

where $C_{L}^{m}(S)$ is the partition of $S$ into components. The best-known solution for graph games is the Myerson value $\mu$ which is obtained by assigning to every graph game $(v, L)$ the Shapley value of the restricted game $v^{L}$, i.e.,

$$
\mu(v, L)=S h\left(v^{L}\right) \text { for all }(v, L) \in \mathcal{G}^{N} \times \mathcal{L}^{N} .
$$

This solution is introduced by Myerson (1977) as the unique solution satisfying component efficiency and fairness (the latter axiom stating that deleting a link between two players changes their payoff by the same amount). Later, Borm, Owen and Tijs (1992) characterized this solution on the class of cycle-free graph games by the following four axioms.

Component efficiency For every graph game $(v, L)$ and every component $C$ of $N$ in $(N, L)$ it holds that $\sum_{i \in C} f_{i}(v, L)=v(C)$.

Additivity For every pair of graph games $(v, L),(w, L)$ it holds that ${ }^{8} f(v+w, L)=$ $f(v, L)+f(w, L)$.

Link $l \in L$ is called superfluous in graph game $(v, L)$ if $v^{E}(N)=v^{E \cup\{l\}}(N)$ for all $E \subseteq L$.

\footnotetext{
${ }^{8}$ For two games $v, w \in \mathcal{G}^{N}$ the sum game is defined by $(v+w)(S)=v(S)+w(S)$ for all $S \subseteq N$.
} 
Superfluous link property If $l \in L$ is a superfluous link in graph game $(v, L)$, then $f(v, L)=f(v, L \backslash\{l\})$.

A graph game $(v, L)$ is called point unanimous if there is a $c^{*} \in \mathbb{R}$ such that $v^{L}(S)=c^{*}$ for all $S \subseteq N$ with $D(N, L) \subseteq S$, and $v^{L}(S)=0$ for all $S \subseteq N$ with $D(N, L) \nsubseteq \subseteq$, i.e., the corresponding restricted game is a multiple of the unanimity game $u_{D(N, L)}$ with respect to the set of non-isolated players $D(N, L)$.

Communication ability property If graph game $(v, L)$ is point unanimous, then there is an $\alpha \in \mathbb{R}$ such that $f_{i}(v, L)=\alpha$ for all $i \in D(N, L)$, and $f_{i}(v, L)=0$ for all $i \in N \backslash D(N, L)$.

Theorem 1 [Borm, Owen and Tijs (1992) $]^{9}$

The Myerson value is the unique solution on the class of cycle-free graph games satisfying component efficiency, additivity, the superfluous link property and the communication ability property.

If the graph $(N, L)$ is a tree (i.e. connected and cycle-free) then we call $(v, L)$ a tree game. For such tree games, Demange (2004) defines for every player $i \in N$ the corresponding hierarchical outcome $h^{i}(v, L)$ as the marginal vector $m^{\pi}\left(v^{L}\right)$ (see (2.1)) of the Myerson restricted game that is obtained for any permutation of the players that is consistent with the tree $L$, i.e. if the path from $i$ to $g$ contains $j$ then $\pi(g)<\pi(j)$. In other words, it is the marginal vector corresponding to a permutation where players enter 'from the bottom' when $i$ is the root. Alternatively, defining $\bar{S}_{j}^{i}(L)=\{g \in N \mid j$ belongs to the path from $i$ to $g\}$ and $S_{j}^{i}(L)=\bar{S}_{j}^{i}(L) \cap R_{(N, L)}(j)$ as the set of subordinates, respectively (direct) successors, of $j$ in the rooted tree with root player $i$, the hierarchical outcomes are also given by

$$
h_{j}^{i}(v, L)=v\left(\bar{S}_{j}^{i}(L)\right)-\sum_{g \in S_{j}^{i}(L)} v\left(\bar{S}_{g}^{i}(L)\right) \text { for all } i, j \in N .
$$

(Note that $j \in \bar{S}_{j}^{i}(L) \backslash S_{j}^{i}(L)$.) Le Breton, Owen and Weber (1992) and Demange (1994) have shown that the Core of the restricted game $v^{L}$ is nonempty if the game $v$ is superadditive and the graph $L$ is cycle-free, respectively, a tree. More specifically, Demange (2004) shows that in case game $v$ is superadditive and $L$ is a tree, then for any $i \in N$ the hierarchical outcome $h^{i}(v, L)$ is an extreme point of Core $\left(v^{L}\right)$. (Note that there can be other extreme points of the Core that are not hierarchical outcomes.) By convexity of the

\footnotetext{
${ }^{9}$ Borm, Owen and Tijs (1992) define a stronger version of the communication ability property using point anonymous games, but as mentioned in van den Brink, van der Laan and Pruzhansky (2004) they only apply the weaker version as stated here.
} 
Core, this implies that all convex combinations of hierarchical outcomes are Core elements. In particular, the average hierarchical outcome of tree game $(v, L)$

$$
A H O(v, L)=\frac{1}{n} \sum_{i \in N} h^{i}(v, L)
$$

belongs to the Core of $v^{L}$. Herings, van der Laan and Talman (2008) extend this definition to cycle-free graph games, by determining the average payoff of a player only over the hierarchical outcomes in its own component ${ }^{10}$, i.e.

$$
\begin{aligned}
A H O_{j}(v, L) & =\frac{1}{\left|K_{L}^{j}\right|} \sum_{i \in K_{L}^{j}} h_{j}^{i}\left(\left.v\right|_{K_{L}^{i}}, L\left(K_{L}^{i}\right)\right) \\
& =\frac{1}{\left|K_{L}^{j}\right|} \sum_{i \in K_{L}^{j}}\left(v\left(\bar{S}_{j}^{i}(L)\right)-\sum_{g \in S_{j}^{i}(L)} v\left(\bar{S}_{g}^{i}(L)\right)\right) \text { for all } j \in N .
\end{aligned}
$$

They define the average tree solution $A T$ as the solution that assigns to every cyclefree graph game its average hierarchical outcome ${ }^{11}$, and provide an axiomatization by component efficiency and an alternative fairness axiom, refered to as component fairness (stating that deleting a link between two players changes the average payoff of the players in the two new components that arise by the same amount.) Moreover, they characterize the average tree solution as the Harsanyi power solution (see van den Brink, van der Laan and Pruzhansky (2004)) given by

$$
A T_{i}(v, L)=\sum_{\substack{T \in C_{L}(N) \\ i \in T}} \frac{1+p_{T}^{L}(i)}{|T|+\sum_{j \in T} p_{T}^{L}(j)} \Delta_{v^{L}}(T), \quad i \in N
$$

where $p_{T}^{L}(j)=\sum_{\substack{g \in N \backslash T \\\{j, g\} \in L}}\left|K_{L \backslash\{j, g\}}^{g}\right|, j \in N$.

\section{An axiomatization of the restricted Banzhaf value for cycle-free graph games}

In this section we consider the restricted Banzhaf value for cycle-free communication graph games that is obtained by applying the Banzhaf value to the restricted game, i.e. we consider the solution $\beta$ given by

$$
\beta(v, L)=B a\left(v^{L}\right) \text { for all }(v, L) \in \mathcal{G}^{N} \times \mathcal{L}^{N} .
$$

\footnotetext{
${ }^{10}$ To be precise, for the first equality we should define solutions also for subgames $\left(K,\left.v\right|_{K}, L(K)\right)$, $K \in C_{L}^{m}(N)$, with $\left.v\right|_{K}(S)=v(S)$ for all $S \subseteq K$, but since we only use these subgames to compute hierarchical outcomes in the cycle-free graph game $(N, v, L)$ we just write graph games as $(v, L)$.

${ }^{11} \mathrm{~A}$ generalization for arbitrary graph games can be found in Herings, van der Laan, Talman and Yang (2008)
} 
Haller (1994) introduced some collusion neutrality properties which state that the sum of payoffs of two players does not change if they 'collude'. He used these properties to axiomatize the Banzhaf value. Later, Malawski (2002) showed that several other collusion neutrality properties can be used ${ }^{12}$. In this paper we consider collusion between two players where they agree to 'act as one' in the sense that they contribute to a coalition only when they both are present ${ }^{13}$. So, when players $i, j \in N, i \neq j$, collude in game $v \in \mathcal{G}^{N}$, then instead of game $v$ we consider the game $v_{i j} \in \mathcal{G}^{N}$ given by

$$
v_{i j}(S)=\left\{\begin{array}{cl}
v(S \backslash\{i, j\}) & \text { if }\{i, j\} \nsubseteq S \\
v(S) & \text { if }\{i, j\} \subseteq S
\end{array}\right.
$$

Applying this idea to communication graph games, it seems reasonable to weaken collusion neutrality by allowing only neighbours in the communication graph to collude. Indeed, it seems counterintuitive that two players who cannot cooperate because they cannot communicate would be able to collude, since collusion is a stronger form of coalition formation then cooperation.

Collusion neutrality If $\{i, j\} \in L$ then $f_{i}\left(v_{i j}, L\right)+f_{j}\left(v_{i j}, L\right)=f_{i}(v, L)+f_{j}(v, L)$, where $v_{i j}$ is given by $(3.5)$.

Next, the isolated player property states that isolated players just earn their singleton worth.

Isolated player property If $R_{(N, L)}(i)=\emptyset$ then $f_{i}(v, L)=v(\{i\})$.

The restricted Banzhaf value is characterized by collusion neutrality, additivity, the superfluous link property, the communication ability property and the isolated player property.

Theorem 2 The restricted Banzhaf value is the unique solution for cycle-free graph games that satisfies collusion neutrality, additivity, the superfluous link property, the communication ability property and the isolated player property.

Proof

It is straightforward to verify that the restricted Banzhaf value satisfies these five axioms. To show uniqueness, suppose that solution $f$ satisfies these five axioms for cycle-free graph games.

\footnotetext{
${ }^{12} \mathrm{~A}$ characterization of the Banzhaf value with collusion properties in terms of inequalities can be found in Lehrer (1988).

${ }^{13}$ This is a collusion game as used by Malawski (2002), but the results of this paper, in particular Theorems 2 and 4, also hold when taking the collusion games of Haller (1994).
} 
First, consider cycle-free graph game $\left(c u_{T}, L\right), c \in \mathbb{R}, c \neq 0, \emptyset \neq T \subseteq N$. If $L=L^{\emptyset}:=\emptyset$, then the isolated player property implies that $f_{i}\left(c u_{T}, L^{\emptyset}\right)=v(\{i\})$ for all $i \in N$. For $L \neq L^{\emptyset}$ we distinguish the following two cases.

(i) If there is no component $C$ in $(N, L)$ such that $T \subseteq C$ then all links are superfluous, and the superfluous link property implies that $f\left(c u_{T}, L\right)=f\left(c u_{T}, L^{\emptyset}\right)$. (Note that in this case $|T| \geq 2$.) Thus, $f_{i}\left(c u_{T}, L\right)=v(\{i\})=0$ for all $i \in N$ as determined above.

(ii) If there is a component $C$ in $(N, L)$ such that $T \subseteq C$ then consider the connected hull $H(T)=\{h \in N \mid$ there exist $i, j \in T$ such that $h$ belongs to the path between $i$ and $j\}$ of $T$. (Note that in a cycle-free graph there is exactly one path between any pair of connected players.) Since $\left(c u_{T}, L(H(T))\right)$ is point unanimous, the communication ability property implies that there is an $\alpha \in \mathbb{R}$ such that $f_{i}\left(c u_{T}, L(H(T))\right)=\alpha$ for all $i \in H(T)$, and $f_{i}\left(c u_{T}, L(H(T))\right)=0$ for all $i \in N \backslash H(T)$. Since all links outside the connected hull of $T$ are superfluous, the superfluous link property implies that

$$
f_{i}\left(c u_{T}, L\right)=f_{i}\left(c u_{T}, L(H(T))\right)= \begin{cases}\alpha & \text { for all } i \in H(T) \\ 0 & \text { for all } i \in N \backslash H(T) .\end{cases}
$$

We prove uniqueness of $f\left(c u_{T}, L\right)$ by induction on $|H(T)|$. If $|H(T)|=1$ then the isolated player property implies that $\alpha=c$, and thus $f\left(c u_{T}, L\right)=\beta\left(c u_{T}, L\right)$ is determined with (3.6) and (2.2).

Proceeding by induction, suppose that $\alpha=\frac{c}{2^{\left|H\left(T^{\prime}\right)\right|-1}}$ has been determined whenever $\left|H\left(T^{\prime}\right)\right|<$ $|H(T)|$. Since $L$ is cycle-free, there is a $j \in T$ such that $\left|R_{(N, L(H(T)))}(j)\right|=1$, i.e. $j$ is a pending player in $(N, L(H(T)))$. Let $h \in R_{(N, L(H(T)))}(j) \cap H(T)$ be the unique neighbour of $j$ in $(N, L(H(T)))$. We distinguish two cases:

Case A. Suppose that $h \in T$. Consider the game $c u_{T \backslash\{j\}}$. Since $H(T \backslash\{j\})=H(T) \backslash\{j\}$, with the induction hypothesis it follows that $f_{h}\left(c u_{T \backslash\{j\}}, L\right)=\frac{c}{2^{|H(T \backslash\{j\})|-1}}=\frac{c}{2^{|H(T)|-2}}$ and $f_{j}\left(c u_{T \backslash\{j\}}, L\right)=0$.

Since $\left(u_{T \backslash\{j\}}\right)_{h j}=u_{T}$, collusion neutrality implies that $f_{h}\left(c u_{T}, L\right)+f_{j}\left(c u_{T}, L\right)=f_{h}\left(c u_{T \backslash\{j\}}, L\right)+$ $f_{j}\left(c u_{T \backslash\{j\}}, L\right)=\frac{c}{2^{|H(T)|-2}}+0=\frac{c}{2^{|H(T)|-2}}$.

By (3.6) and $h, j \in H(T)$ we then have that $\alpha=\frac{c}{2^{|H(T)|-1}}$, and thus $f\left(c u_{T}, L\right)=\beta\left(c u_{T}, L\right)$.

Case B. Suppose that $h \notin T$. Consider the game $c u_{T \cup\{h\}}$. Since $H(T \cup\{h\})=H(T)$, according to Case A, $f\left(c u_{T \cup\{h\}}, L\right)=\beta\left(c u_{T \cup\{h\}}, L\right)$ is determined. Since $c u_{T \cup\{h\}}=\left(c u_{T}\right)_{j h}$, by collusion neutrality we have that $f_{h}\left(c u_{T}, L\right)+f_{j}\left(c u_{T}, L\right)=f_{h}\left(c u_{T \cup\{h\}}, L\right)+f_{j}\left(c u_{T \cup\{h\}}, L\right)=$ $\frac{c}{2^{|H(T)|-2}}$. By (3.6) we again have that $\alpha=\frac{c}{2^{|H(T)|-1}}$, and thus $f\left(c u_{T}, L\right)=\beta\left(c u_{T}, L\right)$.

So, $f\left(c u_{T}, L\right)=\beta\left(c u_{T}, L\right)$ is uniquely determined in both cases. 
Obviously, for the null game given by $v_{0}(S)=0$ for all $S \subseteq N$, the superfluous link property and the isolated player property imply that $f_{i}\left(v_{0}, L\right)=f_{i}\left(v_{0}, L^{\emptyset}\right)=0=\beta_{i}\left(v_{0}, L\right)$ for all $i \in N$.

Finally, since every $v \in \mathcal{G}^{N}$ can be written as a linear combination of unanimity games in a unique way as $v=\sum_{\substack{T \subseteq N \\ T \neq \emptyset}} \Delta_{v}(T) u_{T}$, additivity implies that $f_{i}(v, L)=\sum_{\substack{T \subseteq N \\ i \in H(T)}} \frac{\Delta_{v} L(T)}{2^{|H(T)|-1}}=$ $\beta_{i}(v, L), i \in N$, is uniquely determined for all $(v, L) \in \mathcal{G}^{N} \times \mathcal{L}_{C F}^{N}$.

Note that the Myerson value also satisfies the isolated player property. So, the difference in the axiomatizations given in Theorems 1 and 2 is the Myerson value satisfying component efficiency, and the restricted Banzhaf value satisfying collusion neutrality ${ }^{14}$.

\section{Hierarchical outcomes and an axiomatization of the average tree solution for cycle-free graph games}

Both component efficiency and collusion neutrality seem to be desirable properties. For TU-games, van den Brink (2009) has shown that there is no solution satisfying efficiency, collusion neutrality and the null player property. However, when we allow collusion to occur only between neighbours in a cycle-free communication graph, then there are solutions that satisfy component efficiency, collusion neutality and the superfluous player property. A player $i \in N$ is called superfluous in graph game $(v, L)$ if it is a null player in the restricted game, i.e, if $v^{L}(S)=v^{L}(S \backslash\{i\})$ for all $S \subseteq N$. The next axiom is introduced in van den Nouweland (1993) and states that such superfluous players earn zero payoff.

Axiom 1 (Superfluous player property) If $i \in N$ is a superflous player in graph game $(v, L)$ then $f_{i}(v, L)=0$.

In the following, we will often refer to the solution that assigns to every tree game $(v, L)$, the payoff vector $h^{i}(v, L)$ simply as the hierarchical outcome ${ }^{15}$ corresponding to player $i$, or the solution $h^{i}$. It is easy to verify that all hierarchical outcomes satisfy these three axioms for tree games. Moreover, this holds for all their convex combinations. In particular, the average tree solution satisfies these three properties on the class of cycle-free graph games. (The straightforward proof is left for the reader.)

\footnotetext{
${ }^{14}$ Similar as done for games with a hierarchical permision structure in van den Brink (2000), the Myerson value can be characterized using a 'grand' version of collusion neutrality stating that if two neighbours in a graph game collude then the total sum of payoffs over all players does not change.

${ }^{15}$ Similar, in the literature the Core often refers to the set of efficient, group stable payoff vectors of a game, as well as the solution that assigns this set to every game.
} 
Proposition 3 All solutions $h^{i}, i \in N$, satisfy component efficiency, collusion neutrality and the superfluous player property on the class of tree games. The average tree solution satisfies these properties on the class of cycle-free graph games.

Thus, the 'impossibility' concerning the existence of a solution for TU-games satisfying efficiency, collusion neutrality and the null player property is 'solved' when restricting cooperation by allowing only connected coalitions in a cycle-free communication graph to cooperate and only neighbours can collude.

To axiomatize the average tree solution, we further use additivity and two other axioms. First, the equal gain/loss property states that collusion of two players has the same effect on the payoffs of other players ${ }^{16}$. Again, we only allow collusion among neighbours in the communication graph.

Axiom 2 (Equal gain/loss property) If $\{i, j\} \in L$ then $f_{h}\left(v_{i j}, L\right)-f_{h}(v, L)=f_{g}\left(v_{i j}, L\right)-$ $f_{g}(v, L)$ for all $h, g \in N \backslash\{i, j\}$.

Finally, we use the axiom which states that payoffs in one component do not depend on the game in and structure of other components.

Axiom 3 (Component independence) If $v, w \in \mathcal{G}^{N}$ and $L, L^{\prime} \in \mathcal{L}^{N}$ are such that $v(S)=w(S)$ for all $S \subseteq T \in C_{L}^{m}(N) \cap C_{L^{\prime}}^{m}(N)$, and $L(T)=L^{\prime}(T)$, then $f_{i}(v, L)=f_{i}\left(w, L^{\prime}\right)$ for all $i \in T$.

Next, we state an axiomatization of the average tree solution for cycle-free graph games ${ }^{17}$.

Theorem 4 The average tree solution is the unique solution for cycle-free graph games that satisfies component efficiency, collusion neutrality, additivity, the communication ability property, the superfluous player property, the equal gain/loss property and component independence.

\footnotetext{
${ }^{16}$ Note that this also can be seen as some kind of fairness axiom concerning collusion of players.

${ }^{17}$ The hierarchical outcomes and the average tree solution satisfy the no gain/loss property, which is stronger than the equal gain/loss property, and states that after a collusion of two neighbours the payoffs of the other players do not change. After communicating Theorem 4 and this remark by e-mail and in personal communication with Dolf Talman, in Mishra and Talman (2009) it is shown that using this no gain/loss property instead of the equal gain/loss property, we do not need collusion neutrality to characterize the average tree solution for cycle-free graph games. In fact they use a weaker version of the no gain/loss property, refered to as independence in unanimity games, which requires this property only for unanimity games and connected coalitions. Note that, as usual, in the proof of Theorem 4 all axioms, except additivity, are applied only to unanimity games.
} 


\section{PROOF}

Using (2.4) and Proposition 3 it is straightforward to verify that the average tree solution satisfies these seven axioms.

To show uniqueness, suppose that solution $f$ satisfies these seven axioms for cycle-free graph games.

First, consider graph game $\left(c u_{T}, L\right), c \in \mathbb{R}, c \neq 0, \emptyset \neq T \subseteq N$. If $L=L^{\emptyset}=\emptyset$, then component efficiency implies that $f_{i}\left(c u_{T}, L^{\emptyset}\right)=v(\{i\})$ for all $i \in N$. Next, for $L \neq L^{\emptyset}$ we distinguish the same two cases as in the proof of Theorem 2 (which, however, are treated different).

(i) If there is no component $C$ in $(N, L)$ such that $T \subseteq C$ then all players are superfluous, and the superfluous player property implies that $f_{i}\left(c u_{T}, L\right)=0$ for all $i \in N$.

(ii) If there is a component $C$ in $(N, L)$ such that $T \subseteq C$ then component independence implies that $f\left(c u_{T}, L\right)=f\left(c u_{T}, L(C)\right)$. Component efficiency ${ }^{18}$ implies that

$$
f_{i}\left(c u_{T}, L\right)=f_{i}\left(c u_{T}, L(C)\right)=0 \text { for all } i \in N \backslash C .
$$

We prove uniqueness of $f\left(c u_{T}, L\right)$ by induction on $|H(T)|$ (i.e. the number of players in the connected hull of $T$ ), but now starting with $|H(T)|=|C|$. If $|H(T)|=|C|$ then $\left(c u_{T}, L(C)\right)$ is point unanimous, and thus the communication ability property implies that there is an $\alpha \in \mathbb{R}$ such that $f_{i}\left(c u_{T}, L\right)=\alpha$ for all $i \in C$. Component efficiency then determines that $\alpha=\frac{c}{|C|}$, and thus with component independence we have that

$$
f_{i}\left(c u_{T}, L\right)=f_{i}\left(c u_{T}, L(C)\right)=\left\{\begin{array}{cl}
\frac{c}{|C|} & \text { if } i \in C \\
0 & \text { otherwise }
\end{array}\right.
$$

Proceeding by induction, suppose that $f\left(c u_{T^{\prime}}, L\right)$ has been uniquely determined whenever $\left|H\left(T^{\prime}\right)\right|>|H(T)|$. Since $H(T) \neq C$, there exists a $h \in H(T)$ such that $R_{(N, L)}(h) \backslash H(T) \neq$ $\emptyset$. Take such an $h$ and $j \in R_{(N, L)}(h) \backslash H(T)$.

We distinguish the same two cases as in the proof of Theorem 2:

Case A. Suppose that $h \in T$.

Since $j \notin T$, we have that $\left(c u_{T}\right)_{h j}=c u_{T \cup\{j\}}$, and thus collusion neutrality implies that

$$
f_{j}\left(c u_{T}, L\right)+f_{h}\left(c u_{T}, L\right)=f_{j}\left(c u_{T \cup\{j\}}, L\right)+f_{h}\left(c u_{T \cup\{j\}}, L\right) .
$$

The superfluous player property implies that $f_{j}\left(c u_{T}, L\right)=0$.

Since $|H(T \cup\{j\})|=|H(T)|+1$, the payoff vector $f\left(c u_{T \cup\{j\}}, L\right)$ is determined by the induction hypothesis. Therefore, by $(4.8), f_{h}\left(c u_{T}, L\right)=f_{h}\left(c u_{T \cup\{j\}}, L\right)+f_{j}\left(c u_{T \cup\{j\}}, L\right)-$ $f_{j}\left(c u_{T}, L\right)$ is uniquely determined.

\footnotetext{
${ }^{18}$ The same follows from the superfluous player property.
} 
The equal gain/loss property implies that there is a $\beta \in \mathbb{R}$ such that $f_{i}\left(c u_{T}, L\right)=$ $f_{i}\left(c u_{T \cup\{h\}}, L\right)+\beta$ for all $i \in H(T) \backslash\{h\}$. Again, by the induction hypothesis $f\left(c u_{T \cup\{h\}}, L\right)$ is determined. Since $f_{i}\left(c u_{T}, L\right)=0$ for all $i \in N \backslash H(T)$ by the superfluous player property, and $f_{h}\left(c u_{T}, L\right)$ is determined above, component efficiency determines $\beta$. Thus, $f\left(c u_{T}, L\right)$ is determined.

Case B. Suppose that $h \notin T$. Consider the game $c u_{T \cup\{h\}}$. Since $H(T \cup\{h\})=H(T)$, according to Case A, $f\left(c u_{T \cup\{h\}}, L\right)$ is uniquely determined. Since $h \in H(T) \backslash T$ there exist at least two paths $\left(h_{1}, \ldots, h_{p}\right)$ and $\left(z_{1}, \ldots, z_{q}\right)$ such that $h_{1}=z_{1}=h,\left\{h_{p}, z_{q}\right\} \subseteq$ $T,\left\{h_{1}, \ldots, h_{p-1}\right\} \cap T=\emptyset,\left\{z_{1}, \ldots, z_{q-1}\right\} \cap T=\emptyset$ and $\left\{h_{1}, \ldots, h_{p}\right\} \cap\left\{z_{1}, \ldots, z_{q}\right\}=\{h\}$.

First, consider the path $\left(h_{1}, \ldots, h_{p}\right)$. Since $\left(c u_{T \cup\{h\}}\right)_{h h_{2}}=c u_{T \cup\left\{h, h_{2}\right\}}$, collusion neutrality implies that $f_{h}\left(c u_{T \cup\left\{h, h_{2}\right\}}, L\right)+f_{h_{2}}\left(c u_{T \cup\left\{h, h_{2}\right\}}, L\right)=f_{h}\left(c u_{T \cup\{h\}}, L\right)+f_{h_{2}}\left(c u_{T \cup\{h\}}, L\right)$ is determined since $f\left(c u_{T \cup\{h\}}, L\right)$ is determined. Component efficiency and the equal gain loss property ${ }^{19}$ then determine the payoffs $f_{g}\left(c u_{T \cup\left\{h, h_{2}\right\}}, L\right)$ for all $g \in N \backslash\left\{h, h_{2}\right\}$.

Since $\left(c u_{T \cup\left\{h_{2}\right\}}\right)_{h_{2} h}=c u_{T \cup\left\{h, h_{2}\right\}}$, again applying collusion neutrality yields that $f_{h}\left(c u_{T \cup\left\{h_{2}\right\}}, L\right)+$ $f_{h_{2}}\left(c u_{T \cup\left\{h_{2}\right\}}, L\right)=f_{h}\left(c u_{T \cup\left\{h, h_{2}\right\}}, L\right)+f_{h_{2}}\left(c u_{T \cup\left\{h, h_{2}\right\}}, L\right)$ is determined. Again by component efficiency and the equal gain loss property the payoffs $f_{g}\left(c u_{T \cup\left\{h_{2}\right\}}, L\right)$ are determined for all $g \in N \backslash\left\{h, h_{2}\right\}$.

Repeated application of this argument we end up ${ }^{20}$ with game $c u_{T}$, and thus $f_{g}\left(c u_{T}, L\right)$ are determined for all $g \in N \backslash\left\{h_{1}, \ldots, h_{p}\right\}$.

Applying a similar reasoning to the path $\left(z_{1}, \ldots, z_{q}\right)$ yields that $f_{g}\left(c u_{T}, L\right)$ are determined for all $g \in N \backslash\left\{z_{1}, \ldots, z_{q}\right\}$.

So, all payoffs $f_{g}\left(c u_{T}, L\right), g \in\left(N \backslash\left\{h_{1}, \ldots, h_{p}\right\}\right) \cup\left(N \backslash\left\{z_{1}, \ldots, z_{q}\right\}\right)=N \backslash\{h\}$, are determined. Component efficiency then determines $f_{h}\left(c u_{T}, L\right)$.

So, in both cases $f\left(c u_{T}, L\right)$ is uniquely determined.

Obviously, for the null game given by $v_{0}(S)=0$ for all $S \subseteq N$, the superfluous player property implies that $f_{i}\left(v_{0}, L\right)=f_{i}\left(v_{0}, L^{\emptyset}\right)=0$ for all $i \in N$.

Since every arbitrary $v \in \mathcal{G}^{N}$ can be written as a linear combination of unanimity games in a unique way as $v=\sum_{\substack{T \subseteq N \\ T \neq \emptyset}} \Delta_{v}(T) u_{T}$, additivity implies that $f_{i}(v, L)$ is uniquely determined for all $(v, L) \in \mathcal{G}^{N} \times \mathcal{L}_{C F}^{N}$.

From the proof it also follows that deleting component independence from the axioms, characterizes the average tree solution for tree games as considered in Demange (1994,

\footnotetext{
${ }^{19}$ This also follows from the equal gain/loss property and the superfluous player property since player $j$ is superfluous in both games and thus earns zero in both games.

${ }^{20}$ In the last step we apply this argument to $\left(c u_{T}\right)_{h_{p} h_{p-1}}=c u_{T \cup\left\{h_{p-1}\right\}}$.
} 
2004).

Since the set-valued solution that assigns to every cycle-free graph game the Core of the restricted game satisfies component efficiency, the superfluous player property and component independence ${ }^{21}$, as a corollary of Theorem 4 and Demange (2004)'s result that every hierarchical outcome is an extreme point of the Core of the restricted game if $v$ is superadditive and $L$ is cycle-free, and the Core of a game being a convex set, we obtain that the average tree solution is the unique solution for cycle-free graph games that satisfies collusion neutrality, additivity, the communication ability property, the equal gain/loss property and is Core stable for superadditive games ${ }^{22}$.

Corollary 5 The average tree solution is the unique solution $f$ for cycle-free graph games that satisfies collusion neutrality, additivity, the communication ability property, the equal gain/loss property and is such that $f(v, L) \in \operatorname{Core}\left(v^{L}\right)$ for all $(v, L) \in \mathcal{G}_{S}^{N} \times \mathcal{L}_{C F}^{N}$.

\section{ProOF}

Since all positively scaled unanimity games are superadditive, it follows similar as the proof of Theorem 4 that $f\left(c u_{T}, L\right)$ is uniquely determined for all $c>0, \emptyset \neq T \subseteq N$ and $L \in \mathcal{L}_{C F}^{N}$. Next, consider $\left(c u_{T}, L\right)$ with $c<0$. Since $-c u_{T}$ is superadditive and $c u_{T}+\left(-c u_{T}\right)$ is the null game (i.e. $\left(c u_{T}+\left(-c u_{T}\right)\right)(S)=0$ for all $\left.S \subseteq N\right)$, it follows from additivity of $f$ and the fact that $f_{i}\left(c u_{T}+\left(-c u_{T}\right), L\right)=0$ for all $i \in N$ (by Core stability), that $f\left(c u_{T}, L\right)=f\left(c u_{T}+\left(-c u_{T}\right), L\right)-f\left(-c u_{T}, L\right)=-f\left(-c u_{T}, L\right)$ is uniquely determined because $-c u_{T}$ is superadditive.

Obviously, for the null game given by $v_{0}(S)=0$ for all $S \subseteq N$, Core stability implies that $f_{i}\left(v_{0}, L\right)=f_{i}\left(v_{0}, L^{\emptyset}\right)=0$ for all $i \in N$.

Again, additivity then determines $f(v, L)$ for all $v \in \mathcal{G}^{N}$ and $L \in \mathcal{L}_{C F}^{N}$.

From the axioms of Theorem 4, both the Myerson value and the Banzhaf value do not satisfy the equal gain/loss property. However, Theorem 4 can also be stated using the weaker axiom which states that after a collusion as described by $v_{i j}$ in (3.5) between players $i$ and $j$ that are symmetric ${ }^{23}$ in the restricted game, the payoffs of other symmetric players in the same component change by the same amount ${ }^{24}$.

\footnotetext{
${ }^{21}$ Of course, these properties should be defined for set-valued solutions.

${ }^{22}$ A similar corollary was stated by Herings, van der Laan and Talman (2008) with their axiomatization using component fairness.

${ }^{23}$ Players $i, j \in N$ are symmetric in a game $v \in \mathcal{G}^{N}$ if $v(S \cup\{i\})=v(S \cup\{j\})$ for all $S \subseteq N \backslash\{i, j\}$.

${ }^{24}$ This can be seen since in the proof of Theorem 4 we need to apply the equal gain/loss property only to players in $H(T)$. Although the second and third time we apply this axiom to all players in $N \backslash\left\{h, h_{2}\right\}$, we could instead apply the superfluous player property to players outside $H(T)$, and the weak equal gain/loss property to players in $H(T) \backslash\left\{h, h_{2}\right\}$.
} 
Axiom 4 (Weak equal gain/loss property) Let $i, j \in N$ be symmetric players in $v^{L}$ such that $\{i, j\} \in L$. Then $f_{h}\left(v_{i j}, L\right)-f_{h}(v, L)=f_{g}\left(v_{i j}, L\right)-f_{g}(v, L)$ for all $h, g \in K_{L}^{i} \backslash\{i, j\}$ that are symmetric with $i, j$ in $v^{L}$.

Replacing the equal gain/loss property by this weak equal gain/loss property in Theorem 4, the axioms that characterize the average tree solution are also satisfied by the Myerson value (except collusion neutrality) and the restricted Banzhaf value (except component efficiency). Therefore, we obtained comparable axiomatizations of these three solutions, where the Myerson value and the average tree solution have in common that they both satisfy component efficiency, the average tree solution and the restricted Banzhaf value have in common that they both satisfy collusion neutrality, and the Myerson value and the restricted Banzhaf value have in common that they both satisfy the superfluous link property, see Table 1. In this way it is also clear that the average tree solution satisfying both component efficiency and collusion neutrality goes 'at a cost', since it does not satisfy the superfluous link property. However, similar disadvantages can be stated for the Myerson value (which does not satisfy collusion neutrality) and the restricted Banzhaf value (which is not component efficient).

\begin{tabular}{|c||c|c|c|}
\hline & Comp. eff. & Collusion neutr. & Superfl. link prop. \\
\hline \hline Myerson value & + & - & + \\
restricted Banzhaf value & - & + & + \\
average tree solution & + & + & - \\
\hline
\end{tabular}

Table 1: Properties of Myerson value, restricted Banzhaf value and average tree solution for cycle-free graph games

\section{$5 \quad$ A generalization using network power measures}

In van den Brink, van der Laan and Pruzhansky (2004), the communication ability property is generalized allowing the payoffs in point unanimous graph games to be allocated according to any positive symmetric power measure. A power measure is a function $\sigma$ that assigns to any graph $(S, L), S \subseteq N$, a nonnegative vector $\sigma(S, L) \in \mathbb{R}_{+}^{|S|}$, yielding the nonnegative power $\sigma_{i}(S, L)$ of node $i \in S$ in the graph $(S, L) .{ }^{25}$ A power measure is symmetric if for any graph $(S, L), S \subseteq N$, and $i, j \in S$ such that $R_{(S, L)}(i) \backslash\{j\}=R_{(S, L)}(j) \backslash\{i\}$, we

\footnotetext{
${ }^{25}$ So, a power measure on $N$ assigns power values to nodes in all subgraphs on $S \subseteq N$.
} 
have $\sigma_{i}(S, L)=\sigma_{j}(S, L)$. It is positive if for any $(S, L)$, the power of node $i$ is positive if and only if $i$ is non-isolated (and thus node $i$ has zero power if it is isolated).

Given a positive power measure $\sigma$, van den Brink, van der Laan and Pruzhansky (2004) define the corresponding Harsanyi power solution, denoted by $\varphi^{\sigma}$, on the class of all graph games on player set $N$ as the solution that distributes the dividend of any (connected) coalition in the restricted game $v^{L}$ proportional to the $\sigma$-power of the players in the corresponding subgraph, i.e.

$$
\varphi_{i}^{\sigma}(v, L)=v(\{i\})+\sum_{S \in C_{L}(N),|S| \geq 2} \frac{\sigma_{i}(S, L(S))}{\sum_{j \in S} \sigma_{j}(S, L(S))} \Delta_{v^{L}}(S) .
$$

Note that for any positive power measure and connected coalition $S \in C_{L}(N)$ with $|S| \geq 2$ it holds that $\sum_{j \in S} \sigma_{j}(S, L(S))>0$. Moreover, for every nonconnected coalition $S \notin C_{L}(N)$ it holds that $\Delta_{v}(S)=0$, so all Harsanyi power solutions satisfy component efficiency.

The $\sigma$-communication ability property states that in case the graph game is point unanimous, the dividends are allocated proportional to the power values of the players in the graph.

$\sigma$-Communication ability property If $(v, L)$ is point unanimous, then there is $\alpha \in \mathbb{R}$ such that $f(v, L)=\alpha \sigma(N, L)$.

Obviously, we have the communication ability property in case we take the equal power measure that assigns the same positive power value to all players that have at least one neighbour, and assigns value zero to all isolated players.

In a similar way as Theorem 1 is generalized in van den Brink, van der Laan and Pruzhansky (2004), we can generalize Theorems 2 and 4.

Theorem 6 For a positive power measure $\sigma$,

(i) there is a unique solution for cycle-free graph games that satisfies collusion neutrality, additivity, the superfluous link property, the isolated player property and the $\sigma$-communication ability property.

(ii) there is a unique solution for cycle-free graph games that satisfies component efficiency, collusion neutrality, additivity, the superfluous player property, the equal gain/loss property, component independence and the $\sigma$-communication ability property.

In particular, by taking $\sigma$ to be the degree measure we obtain some kind of average treeposition value and restricted Banzhaf-position value ${ }^{26}$.

\footnotetext{
${ }^{26}$ Note that the position value itself does not satisfy collusion neutrality.
} 
Similar as Theorem 4, Theorem 6.(ii) can be modified for tree games by deleting component independence. Then, we obtain axiomatizations of the hierarchical outcomes for tree games by taking appropriate power measures. Let $(N, L)$ be a tree. For player $i \in N$, take the power measure $\sigma^{i}$ (on trees) as the one that assigns for every tree $(N, L)$ positive power to player $i$, and assigns power zero to all other players, i.e.

$$
\sigma_{j}^{i}(S, L(S))= \begin{cases}1 & \text { if } j=i \\ 0 & \text { otherwise. }\end{cases}
$$

Although this is not a positive power measure, we have the following. (The proof is left for the reader.)

Theorem 7 The solution $h^{i}, i \in N$, is the unique solution for tree games that satisfies efficiency, collusion neutrality, additivity, the superfluous player property, the equal gain/loss property and the $\sigma^{i}$-communication ability property.

Again this also yields a characterization using the Core property.

Corollary 8 The solution $h^{i}, i \in N$, is the unique solution $f$ for tree games that satisfies collusion neutrality, additivity, the equal gain/loss property, the $\sigma^{i}$-communication ability property, and is such that $f(v, L) \in \operatorname{Core}\left(v^{L}\right)$ for all $(v, L) \in \mathcal{G}_{S}^{N} \times \mathcal{L}_{C F}^{N}$.

Adding the following property we obtain a characterization of the class of hierarchical outcomes as a corollary.

root communication ability property There exists an $i \in N$ such that $f_{j}(v, L)=0$ for all $j \in N \backslash\{i\}$, whenever $(v, L)$ is point unanimous.

This is some kind of representation property which states that in a point unanimous graph game all payoff goes to one player.

Theorem 9 A solution for tree games is a hierarchical outcome if and only if it satisfies component efficiency, collusion neutrality, additivity, the superfluous player property, the equal gain/loss property and the root communication ability property.

\section{Concluding remarks}

In this paper we gave axiomatizations of the Myerson value, the restricted Banzhaf value and the average tree solution, where the difference between these solutions is with respect to the axioms of component efficiency (satisfied by the Myerson value and the average tree solution) collusion neutrality (satisfied by the restricted Banzhaf value and the average 
tree solution) and the superfluous link property (satisfied by the Myerson value and the restricted Banzhaf value).

As mentioned before, collusion neutrality properties are discussed by Haller (1994) and Malawski (2002) to characterize the (non-efficient) Banzhaf value for TU-games. In van den Brink (2009) it is shown that there is no solution that satisfies efficiency, collusion neutrality and the null player property. In that sense, restricting cooperation by allowing only connected coalitions in a cycle-free communication graph to cooperate and neighbours to collude 'solves' this impossibility.

As argued by Haller (1994), collusion neutrality can be seen as some kind of stability property. According to a solution that satisfies collusion neutrality, players have no incentive to merge or split in separate players. Similar, Le Breton, Owen and Weber (1992) and Demange (1994) have shown that the communication restrictions that arise from cycle-free graphs guarantee the existence of a Core payoff vector in the restricted game when $v$ is superadditive, while Demange (2004) introduced the hierarchical outcomes as extreme points of this Core. This shows that situations in which cooperation among players can be modelled as a cycle-free graph game has appealing properties concerning Core stability and collusion neutrality. Examples of such situations are sequencing games (see, e.g. Curiel, Pederzoli and Tijs (1989)), auction games (see Graham, Marshall and Richard (1990)), dual airport games (see Littlechild and Owen (1973)), river games (see Ambec and Sprumont (2002)) and polluted river games (see Ni and Wang (2007)).

In games with hierarchies, collusion neutrality properties are applied to characterize the Banzhaf value in, e.g. van den Brink (2002) for apex games, van den Brink (2000) for games with a permission structure and in Algaba, Bilbao, van den Brink and JiménezLosada (2004b) for games on antimatroids. In van den Brink (2006) the sets of feasible coalitions arising as connected coalitions from a communication graph (and more specific cycle-free and cycle-complete communication graphs) are characterized by similar properties that define an antimatroid. This motivates to apply antimatroids in studying hierarchies to make comparisons with communication networks, and to further study properties like component efficiency and collusion neutrality for these models. Adding properties to the sets of feasible coalitions lead to other (some well-known) models. For example, the sets of feasible coalitions arising from conjunctive permission structures (see Gilles, owen and van den Brink (1992) and van den Brink and Gilles (1996)) are characterized as those antimatroids that are colsed under intersection (see Algaba, Bilbao, van den Brink and Jiménez-Losada (2004a)), while the sets of connected coalitions in communication graphs that are closed under intersection are exactly those arising from cycle-complete communication graphs (see van den Brink (2006)). In voting, van den Brink, Rusinowska and Steffen (2009) applied collusion neutrality properties for measures of power and success in 
situations where some voters are influenced by other voters (their peers) in casting their vote, i.e. a voter might vote against its own inclination when a sufficient number of its peers cast the same vote.

Another question for further study is the exploration of the classes of solutions determined by Theorem 6, and other classes of solutions that satisfy component efficiency and collusion neutrality. Adding more axioms is very restrictive for TU-games ${ }^{27}$ but, as seen in this paper, yields interesting possibilities for cycle-free graph games.

\section{References}

Algaba, E., J.M. Bilbao, R. van den Brink, And A. Jiménez-Losada (2004a), "Cooperative games on antimatroids," Discrete Mathematics, 282, 1-15

Algaba, E., J.M. Bilbao, R. van den Brink, and A. Jiménez-Losada (2004b), "Axiomatizations of the Banzhaf value for cooperative games on antimatroids", Mathematical Methods of Operations Research, 59, 147-166.

Ambec, S., And Y. Sprumont (2002), "Sharing a river", Journal of Economic Theory 107, 453-462.

BANZHAF, J.F. (1965), "Weighted voting doesn't work: a mathematical analysis", Rutgers Law Review, 19, 317-343.

BÉal S., E. RÉmila And P. Solal (2009), "Rooted-tree solutions for tree games", European Journal of Operational Research, doi:10.1016/j.ejor.2009.07.023.

Bondareva, O. (1963), "Some applications of linear programming methods to the theory of cooperative games", Problemy Kibernetiki, 10, 119-139 (in Russian).

Borm, P., G. Owen And S. TiJs (1992), "On the position value for communication situations", SIAM Journal of Discrete Mathematics, 5, 305-320.

BRINK, R. VAN DEN (2000), "Banzhaf permission values for games with a permission structure", Technical Report 341, Department of Mathematics, University of Texas at Arlington, USA.

BRINK, R.VAn DEN (2002), "The apex power measure for directed networks", Social Choice and Welfare, 19, 845-867.

\footnotetext{
${ }^{27}$ In van den Brink (2009) it is shown that TU-game solutions that satisfy efficiency and collusion neutrality are some kind of proportional solutions that divide $v(N)$ proportional to exogenous weights, where these weights depend on $v(N)$.
} 
BRINK, R. VAN DEN (2006), "On hierarchies and communication", Tinbergen Discussion Paper 06/056-1, Tinbergen Institute and VU University, Amsterdam.

BRINK, R. VAN DEN (2007), "Null players or nullifying players: the difference between the Shapley value and equal division solutions", Journal of Economic Theory, 136, 767-775.

BRINK, R. VAN DEN (2009), "Efficiency and collusion neutrality of solutions for cooperative TU-Games", TI-Discussion paper 09-065/1, Tinbergen Institute, Amsterdam.

BRINK R. VAN DEN, AND R.P. GILles (1996), "Axiomatizations of the conjunctive permission value for games with permission structures", Games and Economic Behavior, $12,113-126$.

Brink, R. van den, G. van der LaAn, and V. Pruzhansky (2004), "Harsanyi power solutions for graph-restricted Games", TI-Discussion paper 09-052/1, Tinbergen Institute, Amsterdam.

Brink, R. van Den, A. Rusinowska, And F. Steffen (2009), "Measuring power and satisfaction in societies with opinion leaders: dictator and opinion leader properties", TI-Discussion paper 04-095/1, Tinbergen Institute, Amsterdam.

Charnes, A., And S.C. Littlechild, S. C. (1975), "On the formation of unions in n-person games", Journal of Economic Theory, 10, 386402.

Curiel, I., G. Pederzoli, and S. TiJs (1989), "Sequencing games", European Journal of Operational Research 40, 344-351.

Demange, G. (1994), "Intermediate preferences and stable coalition structures", Journal of Mathematical Economics, 23, 45-58.

Demange, G. (2004), "On group stability in hierarchies and networks", Journal of Political Economy, 112, 754-778.

Dubey, P., And LS. Shapley (1979), "Mathematical properties of the Banzhaf power index", Mathematics of Operations Research, 4, 99-131.

Gilles R.P., G. OWEn, AND R. VAN DEN BRINK (1992), "Games with permission structures: the conjunctive approach", International Journal of Game Theory, 20, 277293.

GiLlies D.B. (1953), Some theorems on n-person games, Princeton University Press, Princeton, NJ. 
Graham, D.A., Marshall, R.C., Richard, J.F. (1990), "Differential payments within a bidder coalition and the Shapley value", American Economic Review, 80, 493-510.

HALler, H. (1994), "Collusion properties of values", International Journal of Game Theory, 23, 261-281.

HARSANYI, J.C. (1959), "A bargaining model for cooperative $n$-person games", in: Contributions to the Theory of Games IV (A.W. Tucker and R.D. Luce, eds.), Princeton University Press, Princeton, NJ, pp. 325-355.

Harary, F. (1969), Graph Theory, Addison-Wesley Publishig Company, Reading, Massachusetts.

Herings, P.J.J., G. van der LaAn, And A.J.J. TAlman (2008), "The average tree solution for cycle-free graph games", Games and Economic Behavior, 62, 77-92.

Herings, P.J.J., G. van Der LaAn, A.J.J. Talman, And Z. Yang (2008), "The average tree solution for cooperative games with communication structure", forthcoming in Games and Economic Behavior.

Le Breton, M., G. Owen and S. Weber (1992), "Strongly balanced cooperative games", International Journal of Game Theory, 20, 419-427.

LEHRER, E. (1988), "An axiomatization of the Banzhaf value", International Journal of Game Theory, 17, 89-99.

Littlechild, S.C, And G. Owen (1973), "A simple expression for the Shapley value in a special case", Management Science 20, 370-372.

Malawaski, M. (2002), "Equal treatment, symmetry and Banzhaf value axiomatizations", International Journal of Game Theory, 31, 47-67.

Mishra, D., And D. Talman (2009) "A Characterization of the Average Tree Solution for Cycle-free Graph Games", CentER Discussion Paper No. 2009-17.

Myerson, R. B. (1977), "Graphs and cooperation in games", Mathematics of Operations Research, 2, 225-229.

Ni, D., AND Y. WANG (2007), "Sharing a polluted river", Games and Economic Behavior, 60, 176-186.

Nouweland, A. van Den (1993), Games and Graphs in Economic Situations, Ph.D. Dissertation, Tilburg University. 
Owen, G. (1986), "Values of graph-restricted games", SIAM Journal on Algebraic and Discrete Methods, 7, 210-220.

Owen G. , (1975), "Multilinear extensions and the Banzhaf value", Naval Research Logistics Quarterly, 22, 741-750.

Shapley, L.S. (1953), "A value for $n$-person games", in: Contributions to the Theory of Games II (H.W. Kuhn and A.W. Tucker, eds.), Princeton University Press, Princeton, NJ, pp. 307-317.

Shapley, L.S. (1967), "On balanced sets and cores", Naval Research Logistics Quarterly, $14,453-460$. 